\title{
THE 3-PRIMARY CLASSIFYING SPACE OF THE FIBER OF THE DOUBLE SUSPENSION
}

\author{
STEPHEN D. THERIAULT
}

(Communicated by Paul Goerss)

\begin{abstract}
Gray showed that the homotopy fiber $W_{n}$ of the double suspension $S^{2 n-1} \stackrel{E^{2}}{\longrightarrow} \Omega^{2} S^{2 n+1}$ has an integral classifying space $B W_{n}$, which fits in a homotopy fibration $S^{2 n-1} \stackrel{E^{2}}{\longrightarrow} \Omega^{2} S^{2 n+1} \stackrel{\nu}{\longrightarrow} B W_{n}$. In addition, after localizing at an odd prime $p, B W_{n}$ is an $H$-space and if $p \geq 5$, then $B W_{n}$ is homotopy associative and homotopy commutative, and $\nu$ is an $H$-map. We positively resolve a conjecture of Gray's that the same multiplicative properties hold for $p=3$ as well. We go on to give some exponent consequences.
\end{abstract}

\section{INTRODUCTION}

Let $E^{2}: S^{2 n-1} \longrightarrow \Omega^{2} S^{2 n+1}$ be the double suspension. Let $W_{n}$ be its homotopy fiber. Gray [G1] showed that $W_{n}$ has an integral classifying space $B W_{n}$ and there exists an integral homotopy fibration

$$
S^{2 n-1} \stackrel{E^{2}}{\longrightarrow} \Omega^{2} S^{2 n+1} \stackrel{\nu}{\longrightarrow} B W_{n} .
$$

Further, after localizing at an odd prime $p, B W_{n}$ is an $H$-space and if $p \geq 5$, then $\nu$ is an $H$-map, $B W_{n}$ is homotopy associative, and [G2] showed that $B W_{n}$ is also homotopy commutative. Gray conjectured that these stronger multiplicative properties also hold at the prime 3 . The purpose of this paper is to positively resolve this conjecture and to discuss some consequences related to exponents.

Theorem 1.1. Let $p \geq 3$. Then there exists a unique multiplication on $B W_{n}$ such that $\Omega^{2} S^{2 n+1} \stackrel{\nu}{\longrightarrow} B W_{n}$ is an H-map. Further, this multiplication is both homotopy associative and homotopy commutative.

Note that the uniqueness statement in Theorem 1.1 is new, regardless of the prime. The idea behind proving Theorem 1.1 is to alter Gray's $H$-structure on $B W_{n}$ to obtain a new $H$-structure for which $\nu$ is an $H$-map. The uniqueness statement assures that the new $H$-structure is homotopic to Gray's original $H$ structure when $p \geq 5$. The homotopy associativity and homotopy commutativity statements are then shown to be consequences of $\nu$ being an $H$-map, which is a different approach than that of [G1, G2].

The fact that $\nu$ is an $H$-map can be used to show an important and useful exponent property of $B W_{n}$. In [CMN1] it was shown that $p \cdot \pi_{*}\left(W_{n}\right)=0$ by

Received by the editors October 30, 2006.

2000 Mathematics Subject Classification. Primary 55P45; Secondary 55R35.

Key words and phrases. Double suspension, $H$-space, exponent. 
showing that the $p^{t h}$-power map on $W_{n}$ is null homotopic. Theorem 1.2 shows that this statement can be delooped. Note that this statement is new for any $p \geq 3$, although its proof for $p \geq 5$ would certainly have been known to Gray.

Theorem 1.2. Let $p \geq 3$. Then the $p^{\text {th }}$-power map on $B W_{n}$ is null homotopic.

There are additional consequences of Theorem 1.1 for the prime 3 which were already known for $p \geq 5$. Gray [G1] showed that at all primes $p \geq 2$ there is a homotopy fibration

$$
B W_{n} \stackrel{j}{\longrightarrow} \Omega^{2} S^{2 n p+1} \stackrel{\phi}{\longrightarrow} S^{2 n p-1}
$$

with the property that the composite $\Omega^{2} S^{2 n+1} \stackrel{\nu}{\longrightarrow} B W_{n} \stackrel{j}{\longrightarrow} \Omega^{2} S^{2 n p+1}$ is homotopic to $\Omega H$, where $H$ is the $p^{\text {th }}$ James-Hopf invariant. Further, the fact that $\Omega H$ has order $p$ was used to show that $j$ also has order $p$. Thus $j$ lifts to a map $j^{\prime}: B W_{n} \longrightarrow \Omega^{2} S^{2 n p+1}\{p\}$, where $\Omega^{2} S^{2 n+1}\{p\}$ is the homotopy fiber of the $p^{t h}$ power map on $\Omega^{2} S^{2 n+1}$. In [G1] it was shown that if $\nu$ is an $H$-map, then so is $j$, and this was refined in [T] to show that if $\nu$ is an $H$-map and $p \geq 3$, then the lift $j^{\prime}$ can be chosen so that it too is an $H$-map. Previously, it was only known that $\nu$, and therefore $j$ and $j^{\prime}$, were $H$-maps for $p \geq 5$. Theorem 1.1 now implies that these are all $H$-maps for $p=3$ as well.

The multiplicativity of $j^{\prime}$ was used in [T] to prove a factorization of the $p^{t h}$-power map on $\Omega^{2} S^{2 n p+1}$ through the double suspension, delooping a result of Harper $[\mathrm{H}]$. This proof is now also valid for $p=3$. Stated in full, it is as follows.

Theorem 1.3. Let $p \geq 3$. Then there is a self-equivalence $\epsilon: \Omega^{2} S^{2 n+1} \longrightarrow$ $\Omega^{2} S^{2 n+1}$ such that $\phi^{\prime}=\phi \circ \epsilon$ has the following properties:

(a) there is a homotopy fibration

$$
B W_{n} \stackrel{j}{\longrightarrow} \Omega^{2} S^{2 n p+1} \stackrel{\phi^{\prime}}{\longrightarrow} S^{2 n p-1} ;
$$

(b) there is a homotopy commutative diagram

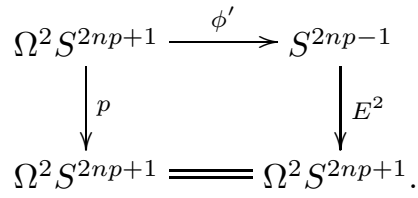

\section{Some Properties of $B W_{n}$}

This section first records some properties of $B W_{n}$ proved by Gray and then builds on them in Lemmas 2.2, 2.3, and 2.5. Beginning integrally, define the space $Y$ and the map $\partial$ by the homotopy fibration sequence

$$
\Omega^{2} S^{2 n+1} \stackrel{\partial}{\longrightarrow} Y \longrightarrow S^{2 n} \stackrel{E}{\longrightarrow} \Omega S^{2 n+1}
$$

where $E$ is the single suspension. In [G1] it was shown that there is a map $Y \stackrel{q}{\longrightarrow} S^{4 n-1}$ with a right homotopy inverse such that the composite $\Omega^{2} S^{2 n+1} \stackrel{\partial}{\longrightarrow}$ $Y \stackrel{q}{\longrightarrow} S^{4 n-1}$ is null homotopic. The space $B W_{n}$ and the map $i$ are defined by the homotopy fibration

$$
B W_{n} \stackrel{i}{\longrightarrow} Y \stackrel{q}{\longrightarrow} S^{4 n-1}
$$


The null homotopy for $q \circ \partial$ implies that there is a factorization

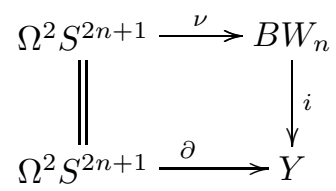

for some map $\nu$. The homotopy fiber of $\nu$ is $S^{2 n-1}$ and it maps into $\Omega^{2} S^{2 n+1}$ by $E^{2}$. The following theorem proved in [G1] will be pivotal.

Theorem 2.1. The map $\Sigma^{2} \Omega^{2} S^{2 n+1} \stackrel{\Sigma^{2} \nu}{\longrightarrow} \Sigma^{2} B W_{n}$ has a right homotopy inverse, and there is a homotopy equivalence $e: \Sigma^{2} \Omega^{2} S^{2 n+1} \longrightarrow \Sigma^{2}\left(S^{2 n-1} \times B W_{n}\right)$ such that $\left(\Sigma^{2} \nu\right) \circ e$ is homotopic to $\Sigma^{2} \pi_{2}$, where $\pi_{2}$ is the projection onto the second factor.

Next, using the loop structure $\mu$ on $\Omega^{2} S^{2 n+1}$, we can multiply to define the map

$$
E^{2} \cdot 1: S^{2 n-1} \times \Omega^{2} S^{2 n+1} \stackrel{E^{2} \times 1}{\longrightarrow} \Omega^{2} S^{2 n+1} \times \Omega^{2} S^{2 n+1} \stackrel{\mu}{\longrightarrow} \Omega^{2} S^{2 n+1} .
$$

Applying [G1, Proposition 1] to the homotopy fibration sequence $\Omega^{2} S^{2 n+1} \stackrel{\partial}{\longrightarrow}$ $Y \longrightarrow S^{2 n} \stackrel{E}{\longrightarrow} \Omega S^{2 n+1}$ shows that there is a homotopy pushout

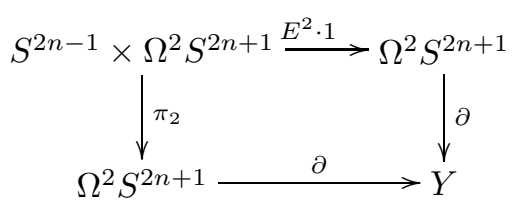

where $\pi_{2}$ is the projection onto the second factor.

Now localize at an odd prime. In [G1] it was shown that there exists a homotopy decomposition $Y \simeq S^{4 n-1} \times B W_{n}$. Thus $\nu$ is homotopic to the composite $\Omega^{2} S^{2 n+1} \stackrel{\partial}{\longrightarrow} Y \stackrel{\pi}{\longrightarrow} B W_{n}$, where $\pi$ is the projection. Composing (2) with this projection immediately gives the following.

Lemma 2.2. Let $p \geq 3$. Then there is a homotopy commutative square

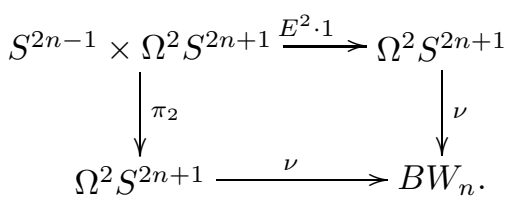

We now turn to new material. The connecting map $\partial$ in the homotopy fibration sequence defining $Y$ determines a canonical homotopy action $\theta: \Omega^{2} S^{2 n+1} \times Y \longrightarrow Y$ with two properties. First, the restrictions of $\theta$ to $\Omega^{2} S^{2 n+1}$ and $Y$ respectively are $\partial$ and the identity map, and second, there is a homotopy commutative square

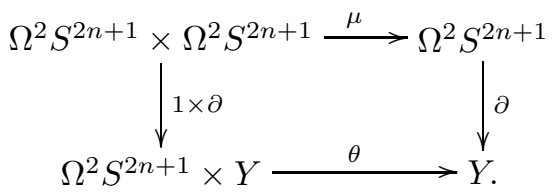


We use this to define an action of $\Omega^{2} S^{2 n+1}$ on $B W_{n}$. The decomposition of $Y$ gives a composite

$$
\bar{\theta}: \Omega^{2} S^{2 n+1} \times B W_{n} \stackrel{1 \times i}{\longrightarrow} \Omega^{2} S^{2 n+1} \times Y \stackrel{\theta}{\longrightarrow} Y \stackrel{\pi}{\longrightarrow} B W_{n} .
$$

Lemma 2.3. Let $p \geq 3$. Then there is a homotopy commutative diagram

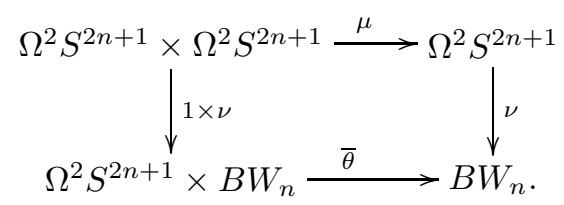

Further, the restrictions of $\bar{\theta}$ to $\Omega^{2} S^{2 n+1}$ and $B W_{n}$ respectively are $\nu$ and the identity map.

Proof. The action of $\Omega^{2} S^{2 n+1}$ on $Y$ gives a homotopy $\theta \circ(1 \times \partial) \simeq \partial \circ \mu$. Compose this with $Y \stackrel{\pi}{\longrightarrow} B W_{n}$. On the one hand, as $\nu \simeq \pi \circ \partial$, we have $\pi \circ \partial \circ \mu \simeq \nu \circ \mu$. On the other hand, as $\partial \simeq i \circ \nu$ by (1), the definition of $\bar{\theta}$ shows that $\pi \circ \theta \circ(1 \times \partial) \simeq$ $\pi \circ \theta \circ(1 \times i) \circ(1 \times \nu) \simeq \bar{\theta} \circ(1 \times \nu)$. Thus $\nu \circ \mu \simeq \bar{\theta} \circ(1 \times \nu)$, proving the homotopy commutativity of the asserted diagram.

As the restrictions of $\theta$ to $\Omega^{2} S^{2 n+1}$ and $Y$ respectively are $\partial$ and the identity map, the definition of $\bar{\theta}$ implies that its restrictions to $\Omega^{2} S^{2 n+1}$ and $B W_{n}$ respectively are $\nu$ and the identity map.

Next, Theorem 2.1 implies that there is a homotopy cofibration

$$
\Sigma^{2}\left(S^{2 n-1} \vee\left(S^{2 n-1} \wedge B W_{n}\right)\right) \stackrel{\alpha}{\longrightarrow} \Sigma^{2} \Omega^{2} S^{2 n+1} \stackrel{\Sigma^{2} \nu}{\longrightarrow} \Sigma^{2} B W_{n}
$$

for some map $\alpha$. In Lemma 2.5 we desuspend in the sense that we construct an explicit map $\Sigma\left(S^{2 n-1} \vee\left(S^{2 n-1} \wedge B W_{n}\right)\right) \longrightarrow \Sigma \Omega^{2} S^{2 n+1}$ whose homotopy cofiber is given by $\Sigma \Omega^{2} S^{2 n+1} \stackrel{\Sigma \nu}{\longrightarrow} \Sigma B W_{n}$.

In general, there is a natural homotopy decomposition $\Sigma A \vee \Sigma B \vee(\Sigma A \wedge B) \stackrel{e}{\longrightarrow}$ $\Sigma(A \times B)$ where

$$
t: \Sigma A \wedge B \hookrightarrow \Sigma A \vee \Sigma B \vee(\Sigma A \wedge B) \stackrel{e}{\longrightarrow} \Sigma(A \times B)
$$

composes trivially with each of the suspended projections $\Sigma \pi_{1}$ and $\Sigma \pi_{2}$. In our case, $t$ can be used to define a Hopf construction on $\Sigma \Omega^{2} S^{2 n+1}$ by the composite

$$
\mu^{*}: \Sigma \Omega^{2} S^{2 n+1} \wedge \Omega^{2} S^{2 n+1} \stackrel{t}{\longrightarrow} \Sigma\left(\Omega^{2} S^{2 n+1} \times \Omega^{2} S^{2 n+1}\right) \stackrel{\Sigma \mu}{\longrightarrow} \Sigma \Omega^{2} S^{2 n+1} .
$$

Lemma 2.2 then has the following useful refinement.

Lemma 2.4. Let $p \geq 3$. Then the composite

$$
\Sigma S^{2 n-1} \wedge \Omega^{2} S^{2 n+1} \stackrel{\Sigma E^{2} \wedge 1}{\longrightarrow} \Sigma \Omega^{2} S^{2 n+1} \wedge \Omega^{2} S^{2 n+1} \stackrel{\mu^{*}}{\longrightarrow} \Sigma \Omega^{2} S^{2 n+1} \stackrel{\Sigma \nu}{\longrightarrow} \Sigma B W_{n}
$$

is null homotopic.

Proof. The naturality of $t$ and the definition of $E^{2} \cdot 1$ give a homotopy commutative diagram

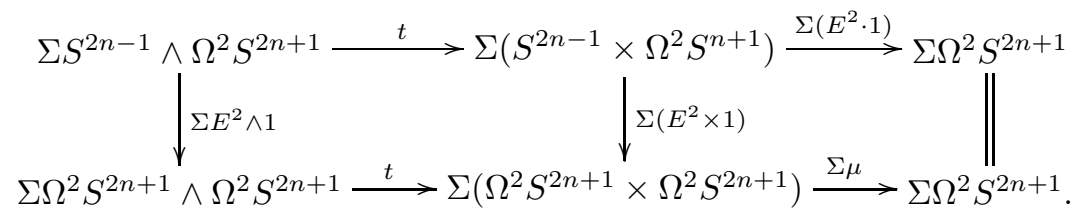


Note that the bottom row is the definition of $\mu^{*}$. Now compose the diagram with the map $\Sigma \Omega^{2} S^{2 n+1} \stackrel{\Sigma \nu}{\longrightarrow} \Sigma B W_{n}$. We obtain $\Sigma \nu \circ \mu^{*} \circ\left(\Sigma E^{2} \wedge 1\right) \simeq \Sigma \nu \circ \Sigma\left(E^{2} \cdot 1\right) \circ t$. By Lemma 2.2, $\nu \circ\left(E^{2} \cdot 1\right) \simeq \nu \circ \pi_{2}$, where $\pi_{2}$ is the projection onto the second factor. As $t$ has the property that $\Sigma \pi_{2} \circ t$ is null homotopic, $\Sigma \nu \circ \Sigma\left(E^{2} \cdot 1\right) \circ t$ is null homotopic. Thus $\Sigma \nu \circ \mu^{*} \circ\left(\Sigma E^{2} \wedge 1\right)$ is null homotopic.

By Theorem 2.1, there is a map $\Sigma^{2} B W_{n} \longrightarrow \Sigma^{2} \Omega^{2} S^{2 n+1}$ which is a right homotopy inverse of $\Sigma^{2} \nu$. Shifting suspension coordinates, we then obtain a composite

$f: \Sigma S^{2 n-1} \wedge B W_{n} \stackrel{\simeq}{\rightrightarrows} S^{2 n-2} \wedge \Sigma^{2} B W_{n} \longrightarrow S^{2 n-1} \wedge \Sigma^{2} \Omega^{2} S^{2 n+1} \stackrel{\simeq}{\rightrightarrows} \Sigma S^{2 n-1} \wedge \Omega^{2} S^{2 n+1}$.

Incorporating this, let $g$ be the composite

$g: \Sigma S^{2 n-1} \wedge B W_{n} \stackrel{f}{\rightarrow} \Sigma S^{2 n-1} \wedge \Omega^{2} S^{2 n+1} \stackrel{\Sigma E^{2} \wedge 1}{\longrightarrow} \Sigma \Omega^{2} S^{2 n+1} \wedge \Omega^{2} S^{2 n+1} \stackrel{\mu^{*}}{\rightarrow} \Sigma \Omega^{2} S^{2 n+1}$.

Denote the wedge sum operation by " $\perp$ ".

Lemma 2.5. Let $p \geq 3$. Then there is a homotopy cofibration

$$
\Sigma S^{2 n-1} \vee\left(\Sigma S^{2 n-1} \wedge B W_{n}\right) \stackrel{\Sigma E^{2} \perp g}{\longrightarrow} \Sigma \Omega^{2} S^{2 n+1} \stackrel{\Sigma \nu}{\longrightarrow} \Sigma B W_{n} .
$$

Proof. We first observe that $\Sigma \nu \circ\left(\Sigma E^{2} \perp g\right)$ is null homotopic. A map out of a wedge is determined by its restriction to each wedge summand, so it is equivalent to show that both $\Sigma \nu \circ \Sigma E^{2}$ and $\Sigma \nu \circ g$ are null homotopic. The former is null homotopic because $\nu$ and $E^{2}$ are consecutive maps in a homotopy fibration. The latter is null homotopic because $g$ is defined as $\mu^{*} \circ \Sigma\left(E^{2} \wedge 1\right) \circ f$ and Lemma 2.2 says that the composite $\Sigma \nu \circ \mu^{*} \circ\left(\Sigma E^{2} \wedge 1\right)$ is null homotopic.

Let $X$ be the homotopy cofiber of $\Sigma E^{2} \perp g$. The null homotopy for the composite $\Sigma \nu \circ\left(\Sigma E^{2} \perp g\right)$ results in an extension

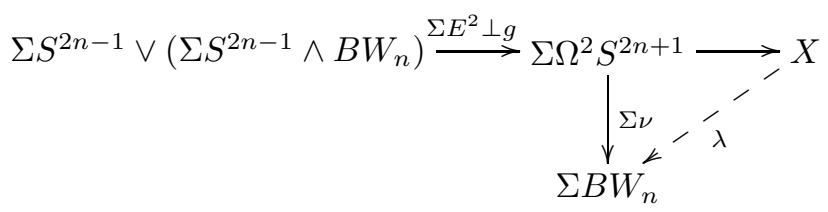

for some map $\lambda$. We claim that $\lambda$ is an isomorphism in homology. If so, then it is a homotopy equivalence and the lemma follows.

By Theorem 2.1, there is a homotopy equivalence

$$
\Sigma^{2} \Omega^{2} S^{2 n+1} \simeq \Sigma^{2}\left(S^{2 n-1} \times B W_{n}\right)
$$

in which $\Sigma^{2} \nu$ becomes the suspended projection $\Sigma^{2} \pi_{2}$. In mod- $p$ homology this implies that $H_{*}\left(\Omega^{2} S^{2 n+1}\right) \cong H_{*}\left(S^{2 n-1} \times B W_{n}\right) \cong H_{*}\left(S^{2 n-1}\right) \otimes H_{*}\left(B W_{n}\right)$ and $\nu_{*}$ is the projection onto $H_{*}\left(B W_{n}\right)$. Further, $\left(E^{2}\right)_{*}$ is the inclusion of $H_{*}\left(S^{2 n-1}\right)$. Therefore, as a module map, $\nu_{*}$ has a right inverse $s: H_{*}\left(B W_{n}\right) \longrightarrow H_{*}\left(\Omega^{2} S^{2 n+1}\right)$ and the product map $H_{*}\left(S^{2 n-1}\right) \otimes H_{*}\left(B W_{n}\right) \stackrel{\left(E^{2}\right)_{*} \cdot s}{\longrightarrow} H_{*}\left(\Omega^{2} S^{2 n+1}\right)$ is an isomorphism. Let $M$ be the submodule of $\Sigma\left(H_{*}\left(S^{2 n-1}\right) \otimes H_{*}\left(B W_{n}\right)\right)$ consisting of elements of the form $\sigma(x \otimes y)$ where both $x$ and $y$ have degree $\geq 1$. The right homotopy inverse for $\Sigma^{2} \nu$ geometrically realizes $\Sigma^{2} s$ and so $g$ geometrically realizes the restriction of $\Sigma\left(\left(E^{2}\right)_{*} \cdot s\right)$ to the submodule $M$. Thus $\left(\Sigma E^{2} \perp g\right)$ geometrically realizes the restriction of $\Sigma\left(\left(E^{2}\right)_{*} \cdot s\right)$ to $H_{*}\left(S^{2 n-1}\right) \oplus M$. The cokernel of $\left(\Sigma E^{2} \perp g\right)_{*}$ is therefore given by the map $\Sigma H_{*}\left(\Omega^{2} S^{2 n+1}\right) \stackrel{\Sigma \nu_{*}}{\longrightarrow} \Sigma H_{*}\left(B W_{n}\right)$. On the other hand, the 
homotopy cofibration defining $X$ says that cokernel of $\left(\Sigma E^{2} \perp g\right)_{*}$ is given by the map $H_{*}\left(\Sigma \Omega^{2} S^{2 n+1}\right) \longrightarrow H_{*}(X)$. Thus $\lambda_{*}$ is an isomorphism.

\section{An $H$-STRuCTuRe ON $B W_{n}$}

In Lemma 2.3 it was shown that there is a homotopy action $\Omega^{2} S^{2 n+1} \times B W_{n} \stackrel{\bar{\theta}}{\longrightarrow}$ $B W_{n}$. In Proposition 3.2 we will show that this action factors through a multiplication on $B W_{n}$. The bulk of the work comes in a preliminary lemma, which proves a suspended factorization.

Lemma 3.1. Let $p \geq 3$. Then there is a homotopy commutative diagram

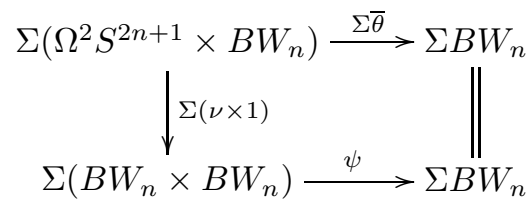

for some map $\psi$ with the property that its restriction to $\Sigma\left(B W_{n} \vee B W_{n}\right)$ is homotopic to the suspension of the folding map.

Proof. As the proof is lengthy, we give an outline. The idea is to decompose $\Sigma\left(\Omega^{2} S^{2 n+1} \times B W_{n}\right)$ as $\Sigma \Omega^{2} S^{2 n+1} \vee \Sigma B W_{n} \vee\left(\Sigma \Omega^{2} S^{2 n+1} \wedge B W_{n}\right)$ and show the corresponding factorization exists for each wedge summand. The factorizations for the first two summands will easily follow from the properties of $\bar{\theta}$. The factorization for the smash product is harder. We use the homotopy cofibration in Lemma 2.5, smashed with $B W_{n}$. This means we need certain null homotopies for the two-term smash product $S^{2 n-1} \wedge B W_{n}$ and the three-term smash product $S^{2 n-1} \wedge B W_{n} \wedge B W_{n}$. The latter is made more complicated as we have to control the map $g$ in Lemma 2.5, which is defined using a Hopf construction, and this will involve some reassociating.

We now begin the proof. Consider the natural homotopy decomposition

$$
e: \Sigma \Omega^{2} S^{2 n+1} \vee \Sigma B W_{n} \vee \Sigma\left(\Omega^{2} S^{2 n+1} \wedge B W_{n}\right) \longrightarrow \Sigma\left(\Omega^{2} S^{2 n+1} \times B W_{n}\right) .
$$

The composition $\Sigma(\nu \times 1) \circ e$ is homotopic to the wedge sum $\Sigma \nu \vee \Sigma 1 \vee \Sigma(\nu \wedge 1)$. So to prove the homotopy commutativity of the diagram asserted by the lemma, it is equivalent to show that $\Sigma \bar{\theta} \circ e$ factors through $\Sigma \nu \vee \Sigma 1 \vee \Sigma(\nu \wedge 1)$. Such a factorization produces a map

$$
\psi^{\prime}: \Sigma B W_{n} \vee \Sigma B W_{n} \vee \Sigma\left(B W_{n} \wedge B W_{n}\right) \longrightarrow \Sigma B W_{n},
$$

which lets us define $\psi$ as the composite

$$
\psi: \Sigma\left(B W_{n} \times B W_{n}\right) \stackrel{\simeq}{\longrightarrow} \Sigma B W_{n} \vee \Sigma B W_{n} \vee \Sigma\left(B W_{n} \wedge B W_{n}\right) \stackrel{\psi^{\prime}}{\longrightarrow} \Sigma B W_{n} .
$$

The additional assertion that $\psi$ can be chosen so its restriction to $\Sigma\left(B W_{n} \vee B W_{n}\right)$ is homotopic to the suspension of the fold map is equivalent to the assertion that $\psi^{\prime}$ can be chosen so that its restriction to $\Sigma B W_{n} \vee \Sigma B W_{n}$ is homotopic to the fold map.

By Lemma 2.3, the restrictions of $\bar{\theta}$ to $\Omega^{2} S^{2 n+1}$ and $B W_{n}$ respectively are $\nu$ and 1. Thus the restrictions of $\Sigma \bar{\theta} \circ$ e to $\Sigma \Omega^{2} S^{2 n+1}$ and $\Sigma B W_{n}$ respectively are $\Sigma \nu$ and $\Sigma 1$. Therefore we can define $\psi^{\prime}$ on the wedge summands $\Sigma B W_{n} \vee \Sigma B W_{n}$ as the folding map. 
It remains to show the factorization of $\Sigma\left(\Omega^{2} S^{2 n+1} \wedge B W_{n}\right)$ through $\Sigma(\nu \wedge 1)$. We break this into three steps.

Step 1: Setting up. In all that follows, a map labelled with a " $t$ " will be an instance of the natural inclusion $\Sigma A \wedge B \stackrel{t}{\longrightarrow} \Sigma(A \times B)$ which composes trivially with $\Sigma \pi_{1}$ and $\Sigma \pi_{2}$. In particular, let $t$ be the map

$$
t: \Sigma \Omega^{2} S^{2 n+1} \wedge B W_{n} \longrightarrow \Sigma\left(\Omega^{2} S^{2 n+1} \times B W_{n}\right) .
$$

We need to show that $(\Sigma \bar{\theta}) \circ t$ factors through $\Sigma(\nu \wedge 1)$. Smashing the homotopy cofibration in Lemma 2.5 and distributing the smash product across the wedge, we obtain a homotopy cofibration

$\left(\Sigma S^{2 n-1} \wedge B W_{n}\right) \vee\left(\Sigma S^{2 n-1} \wedge B W_{n} \wedge B W_{n}\right) \stackrel{\gamma}{\rightarrow} \Sigma \Omega^{2} S^{2 n+1} \wedge B W_{n} \stackrel{\Sigma \nu \wedge 1}{\longrightarrow} \Sigma B W_{n} \wedge B W_{n}$ where $\gamma=\left(\Sigma E^{2} \wedge 1\right) \vee(g \wedge 1)$. So to show that $(\Sigma \bar{\theta}) \circ t$ factors through $\Sigma(\nu \wedge 1)$ it is equivalent to show that both $(\Sigma \bar{\theta}) \circ t \circ\left(\Sigma E^{2} \wedge 1\right)$ and $(\Sigma \bar{\theta}) \circ t \circ(g \wedge 1)$ are null homotopic.

Step 2: The null homotopy for $(\Sigma \bar{\theta}) \circ t \circ\left(\Sigma E^{2} \wedge 1\right)$. Consider the diagram

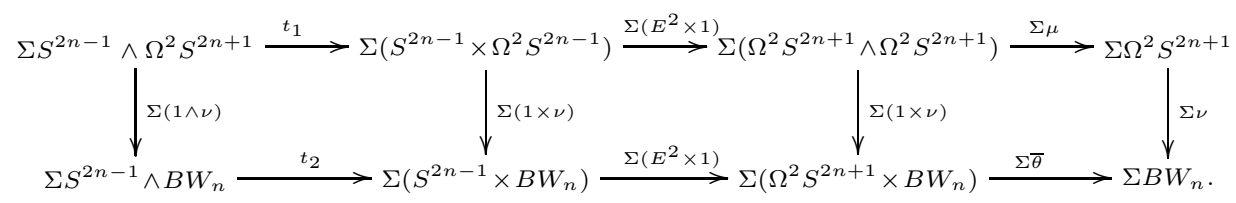

The left square homotopy commutes by naturality, the middle square clearly homotopy commutes, and the right square homotopy commutes by Lemma 2.3. The upper direction around the diagram is $\Sigma \nu \circ \Sigma \mu \circ \Sigma\left(E^{2} \times 1\right) \circ t_{1}$. By definition, $E^{2} \cdot 1=\mu \circ\left(E^{2} \times 1\right)$, so by Lemma $2.2, \nu \circ \mu \circ\left(E^{2} \times 1\right) \simeq \nu \circ \pi_{2}$. As $\left(\Sigma \pi_{2}\right) \circ t_{1}$ is null homotopic, we therefore have $\Sigma \nu \circ \Sigma \mu \circ \Sigma\left(E^{2} \times 1\right) \circ t_{1} \simeq \Sigma \nu \circ \Sigma \pi_{2} \circ t_{1}$ null homotopic. Now consider the lower direction around the diagram. The naturality of the " $t$ "maps implies that $\Sigma\left(E^{2} \times 1\right) \circ t_{2} \simeq t \circ\left(\Sigma E^{2} \wedge 1\right)$. Thus $\Sigma \bar{\theta} \circ \Sigma\left(E^{2} \times 1\right) \circ t_{2} \circ \Sigma(1 \wedge \nu) \simeq$ $\Sigma \bar{\theta} \circ t \circ\left(\Sigma E^{2} \wedge 1\right) \circ \Sigma(1 \wedge \nu)$. The homotopy between the upper and lower directions around the diagram then implies that $\Sigma \bar{\theta} \circ t \circ\left(\Sigma E^{2} \wedge 1\right) \circ \Sigma(1 \wedge \nu)$ is null homotopic. By Theorem 2.1, $1 \wedge \nu$ has a right homotopy inverse and so $\Sigma \bar{\theta} \circ t \circ\left(\Sigma E^{2} \wedge 1\right)$ is null homotopic.

Step 3: The null homotopy for $(\Sigma \bar{\theta}) \circ t \circ(g \wedge 1)$. To compress notation, let $M=$ $\Omega^{2} S^{2 n+1}$. Consider the diagram

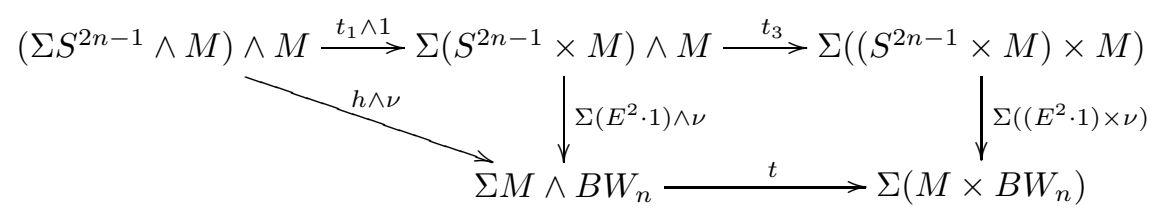

where $h$ is defined as the composite $\Sigma S^{2 n-1} \wedge M \stackrel{\Sigma E^{2} \wedge 1}{\longrightarrow} \Sigma M \wedge M \stackrel{\mu^{*}}{\longrightarrow} \Sigma M$. By definition, $E^{2} \cdot 1=\mu \circ\left(E^{2} \times 1\right)$ and $\mu^{*}=\Sigma \mu \circ t_{4}$, for $t_{4}: \Sigma M \wedge M \longrightarrow \Sigma(M \times M)$. The naturality of the " $t$ "-maps implies that $\Sigma\left(E^{2} \times 1\right) \circ t_{1} \simeq \mu^{*} \circ\left(\Sigma E^{2} \wedge 1\right)=h$ and so the left triangle homotopy commutes. The right square homotopy commutes by the naturality of the " $t$ "-maps. Let $s=t_{3} \circ\left(t_{1} \wedge 1\right)$ be the composite along the top row. Observe that $s$ is trivial when composed with $\Sigma\left(\pi_{2} \times \pi_{3}\right)$, the suspension of 
the product of the projections onto the second and third factors. Now consider the diagram

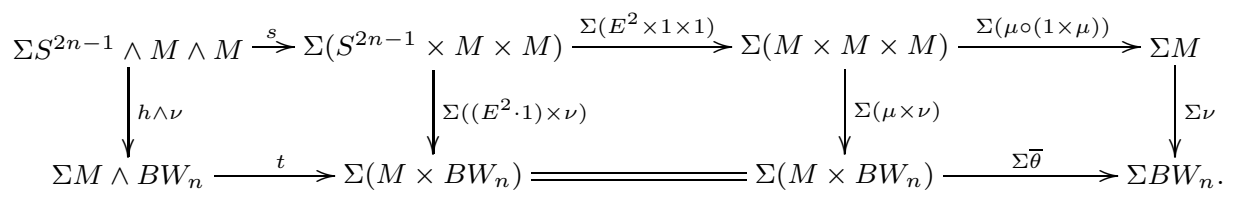

The left square homotopy commutes by the previous diagram, and the middle square homotopy commutes as $E^{2} \cdot 1$ is defined as $\mu \circ\left(E^{2} \times 1\right)$. The right square homotopy commutes because Lemma 2.3 shows that $\bar{\theta} \circ(1 \times \nu) \simeq \nu \circ \mu$ and so, using the homotopy associativity of $M$, we obtain a string of homotopies $\bar{\theta} \circ(\mu \times \nu)=$ $\bar{\theta} \circ(1 \times \nu) \circ(\mu \times 1) \simeq \nu \circ \mu \circ(\mu \times 1) \simeq \nu \circ \mu \circ(1 \times \mu)$. Consider the upper direction around the diagram. To distinguish maps, let $1_{S}$ and $1_{M}$ be the identity maps on $S^{2 n-1}$ and $M$ respectively. In the following,

$$
\begin{aligned}
\nu \circ \mu \circ\left(1_{M} \times \mu\right) \circ\left(E^{2} \times 1_{M} \times 1_{M}\right) & \simeq \nu \circ \mu \circ\left(E^{2} \times \mu\right) \\
& \simeq \nu \circ \mu \circ\left(E^{2} \times 1_{M}\right) \circ\left(1_{S} \times \mu\right) \\
& \simeq \nu \circ \pi_{2} \circ\left(1_{S} \times \mu\right) \\
& \simeq \nu \circ \mu \circ\left(\pi_{2} \times \pi_{3}\right),
\end{aligned}
$$

the first two homotopies reorganize the composites, the third is due to Lemma 2.2, and the fourth uses the naturality of projections. Now suspend and precompose with $s$. As $\Sigma\left(\pi_{2} \times \pi_{3}\right) \circ s$ is null homotopic, the upper direction around the diagram, $\Sigma \nu \circ \Sigma\left(\mu \circ\left(1_{M} \times \mu\right)\right) \circ \Sigma\left(E^{2} \times 1_{M} \times 1_{M}\right) \circ s$, is therefore null homotopic. Hence the lower direction around the diagram, $\Sigma \bar{\theta} \circ t \circ(h \wedge \nu)$, is null homotopic.

Just before Lemma 2.5 we defined a map $\Sigma S^{2 n-1} \wedge B W_{n} \stackrel{f}{\longrightarrow} S^{2 n-1} \wedge M$ which is a left homotopy inverse of $\Sigma\left(1_{S} \wedge \nu\right)$. Also recall that the map $\Sigma S^{2 n-1} \wedge B W_{n} \stackrel{g}{\longrightarrow} \Sigma M$ was defined as $g=\mu^{*} \circ\left(\Sigma E^{2} \wedge 1\right) \circ f$, and so $g=h \circ f$. Let $k$ be the composite

$$
\begin{aligned}
k: \Sigma S^{2 n-1} \wedge B W_{n} \wedge B W_{n} \stackrel{T \wedge 1}{\longrightarrow} B W_{n} \wedge \Sigma S^{2 n-1} \wedge B W_{n} \stackrel{1 \wedge f}{\longrightarrow} \\
B W_{n} \wedge \Sigma S^{2 n-1} \wedge M \stackrel{T \wedge 1}{\longrightarrow} \Sigma S^{2 n-1} \wedge B W_{n} \wedge M,
\end{aligned}
$$

where $T$ is the interchange map. Consider the diagram

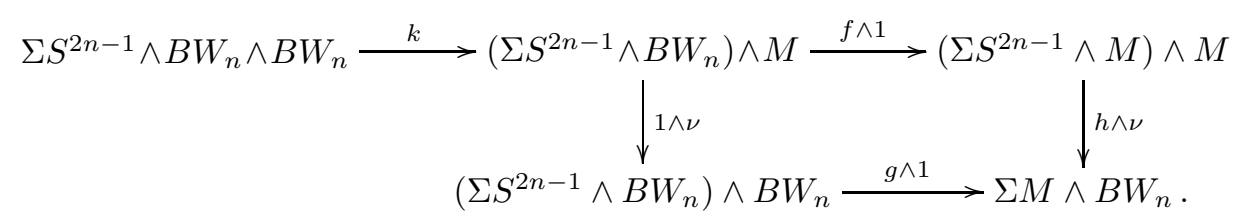

The square homotopy commutes as $g=h \circ f$. Since $f$ is a left homotopy inverse of $\Sigma\left(1_{S} \wedge \nu\right)$, the composite $(1 \wedge \nu) \circ k$ in the lower direction around the diagram is homotopic to the identity map. Thus $(h \wedge \nu) \circ(f \wedge 1) \circ k \simeq g \wedge 1$. Therefore the null homotopy for $\Sigma \bar{\theta} \circ t \circ(h \wedge \nu)$ in the previous paragraph implies that $\Sigma \bar{\theta} \circ t \circ(g \wedge 1)$ is null homotopic, as required. 
Proposition 3.2. Let $p \geq 3$. Then there is an $H$-structure $m: B W_{n} \times B W_{n} \longrightarrow$ $B W_{n}$ which satisfies a homotopy commutative diagram

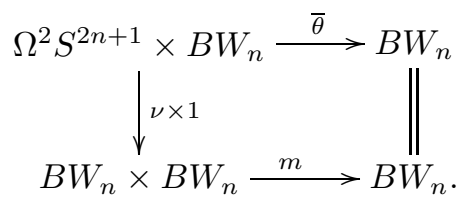

Proof. Since $B W_{n}$ is an $H$-space, the canonical suspension $B W_{n} \stackrel{E}{\longrightarrow} \Omega \Sigma B W_{n}$ has a left homotopy inverse $r: \Omega \Sigma B W_{n} \longrightarrow B W_{n}$. Consider the diagram

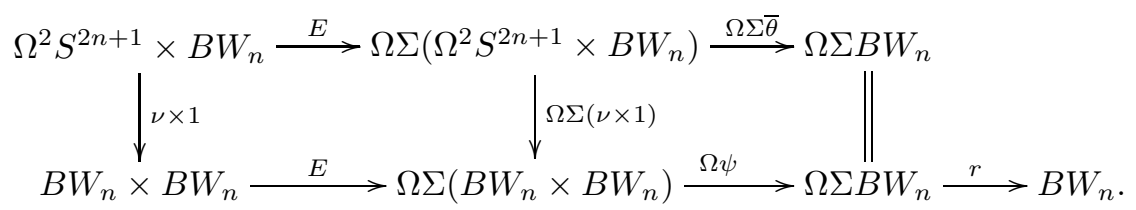

The left square homotopy commutes by the naturality of $E$, and the right square homotopy commutes by Lemma 3.1. Along the top row, the naturality of $E$ implies that $\Omega \Sigma \bar{\theta} \circ E \simeq E \circ \bar{\theta}$, where the suspension $E$ on the right side of the homotopy is $B W_{n} \stackrel{E}{\longrightarrow} \Omega \Sigma B W_{n}$. Thus the upper direction around the diagram satisfies

$$
r \circ \Omega \Sigma \bar{\theta} \circ E \simeq r \circ E \circ \bar{\theta} \simeq \bar{\theta} .
$$

Let $m: B W_{n} \times B W_{n} \longrightarrow B W_{n}$ be the composite $r \circ \Omega \psi \circ E$ along the bottom row. The homotopy commutativity of the diagram implies that $\bar{\theta} \simeq m \circ(\nu \times 1)$, as asserted by the proposition. Finally, since the restriction of $\psi$ to $\Sigma\left(B W_{n} \vee B W_{n}\right)$ is homotopic to the suspension of the folding map, it follows that the restriction of $m$ to $B W_{n} \vee B W_{n}$ is homotopic to the folding map, and so $m$ is an $H$-structure.

Juxtaposing the homotopy commutative diagrams in Lemma 2.3 and Proposition 3.2 gives a homotopy commutative diagram

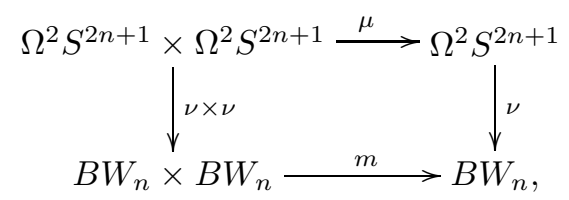

which immediately proves the following.

Corollary 3.3. Let $p \geq 3$. Then the map $\Omega^{2} S^{2 n+1} \stackrel{\nu}{\longrightarrow} B W_{n}$ is an $H$-map with respect to the loop multiplication on $\Omega^{2} S^{2 n+1}$ and the multiplication $m$ on $B W_{n}$.

\section{Properties of the $H$-Structure $m$ on $B W_{n}$}

In this section we use the multiplication $m$ on $B W_{n}$ in Proposition 3.2 to prove Theorems 1.1 and 1.2. We begin with a general lemma, which will get used repeatedly.

Lemma 4.1. Suppose there exist maps $f: X \longrightarrow Y$ and $g, h: Y \longrightarrow Z$ such that $g \circ f \simeq h \circ f, \Sigma^{2} f$ has a right homotopy inverse, and $Z$ is an $H$-space. Then $g \simeq h$. 
Proof. As $Z$ is an $H$-space, there is a map $r: \Omega \Sigma Z \longrightarrow Z$ which is a left homotopy inverse of the suspension $E: Z \longrightarrow \Omega \Sigma Z$. Thus it suffices to show that $\Sigma g \simeq \Sigma h$, for then the naturality of $E$ with respect to loop suspensions implies that there is a string of homotopies $g \simeq r \circ E \circ g \simeq r \circ \Omega \Sigma g \circ E \simeq r \circ \Omega \Sigma h \circ E \simeq r \circ E \circ h \simeq h$.

Define the space $C$ and the map $t$ by the homotopy cofibration

$$
X \stackrel{f}{\longrightarrow} Y \stackrel{t}{\longrightarrow} C .
$$

Since $\Sigma^{2} f$ has a left homotopy inverse, there is a homotopy equivalence $\Sigma^{2} X \simeq$ $\Sigma C \vee \Sigma^{2} Y$. This implies that $\Sigma t$ is null homotopic. The hypothesis $g \circ f \simeq h \circ f$ implies that $\Sigma g \circ \Sigma f \simeq \Sigma h \circ \Sigma f$, and so, using the co- $H$ structure to subtract and distribute, we have $(\Sigma g-\Sigma h) \circ \Sigma f$ null homotopic. Thus there is an extension

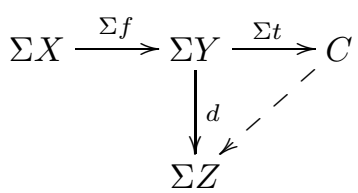

where $d=\Sigma g-\Sigma h$. The null homotopy for $\Sigma t$ therefore implies that $\Sigma g-\Sigma h$ is null homotopic, and so $\Sigma g \simeq \Sigma h$, as required.

We now move on to the assertions of Theorem 1.1, taken one at a time.

Lemma 4.2. There is a unique multiplication on $B W_{n}$ for which $\Omega^{2} S^{2 n+1} \stackrel{\nu}{\longrightarrow}$ $B W_{n}$ is an H-map.

Proof. Let $\mu$ be the loop multiplication on $\Omega^{2} S^{2 n+1}$. Suppose

$$
\mu_{1}, \mu_{2}: B W_{n} \times B W_{n} \longrightarrow B W_{n}
$$

are both multiplications for which $\nu$ is an $H$-map. Then $\mu_{1} \circ(\nu \times \nu) \simeq \nu \circ \mu \simeq$ $\mu_{2} \circ(\nu \times \nu)$. By Theorem 2.1, $\Sigma^{2} \nu$ has a right homotopy inverse, and therefore so does $\Sigma^{2}(\nu \times \nu)$. Summarizing, we have maps $\Omega^{2} S^{2 n+1} \times \Omega^{2} S^{2 n+1} \stackrel{\nu \times \nu}{\longrightarrow} B W_{n} \times B W_{n}$ and $B W_{n} \times B W_{n} \stackrel{\mu_{1}, \mu_{2}}{\longrightarrow} B W_{n}$ such that $\mu_{1} \circ(\nu \times \nu) \simeq \mu_{2} \circ(\nu \times \nu), \Sigma^{2}(\nu \times \nu)$ has a right homotopy inverse, and $B W_{n}$ is an $H$-space. So by Lemma 4.1, $\mu_{1} \simeq \mu_{2}$.

Lemma 4.3. Suppose $B W_{n}$ has an $H$-structure for which $\Omega^{2} S^{2 n+1} \stackrel{\nu}{\longrightarrow} B W_{n}$ is an H-map. Then this H-structure is both homotopy associative and homotopy commutative.

Proof. Let $\mu$ be the loop structure on $\Omega^{2} S^{2 n+1}$, and let $m$ be the $H$-structure on $B W_{n}$ for which $\nu$ is an $H$-map. First consider homotopy associativity. Let $g=m \circ(m \times 1)$ and $h=m \circ(1 \times m)$. Since $\Omega^{2} S^{2 n+1}$ is homotopy associative and $\nu$ is an $H$-map, we have $g \circ(\nu \times \nu \times \nu) \simeq h \circ(\nu \times \nu \times \nu)$. By Theorem 2.1, $\Sigma^{2} \nu$ has a right homotopy inverse, and therefore so does $\Sigma^{2}(\nu \times \nu \times \nu)$. Summarizing, let $X=\Omega^{2} S^{2 n+1} \times \Omega^{2} S^{2 n+1} \times \Omega^{2} S^{2 n+1}, Y=B W_{n} \times B W_{n} \times B W_{n}, Z=B W_{n}$, and $f=\nu \times \nu \times \nu$. Then we have maps $X \stackrel{f}{\longrightarrow} Y$ and $Y \stackrel{g, h}{\longrightarrow} Z$ such that $g \circ f \simeq h \circ f$, $\Sigma^{2} f$ has a right homotopy inverse, and $Z$ is an $H$-space. So by Lemma $4.1, g \simeq h$. That is, $m \circ(m \times 1) \simeq m \circ(1 \times m)$ and so $m$ is homotopy associative.

A similar argument holds for homotopy commutativity by taking $f=\nu \times \nu$, $g=m$, and $h=m \circ T$ where $T$ is the map which interchanges factors.

Proof of Theorem 1.1. Combine the statements of Proposition 3.2, Corollary 3.3 and Lemmas 4.2 and 4.3 . 
Finally, we prove the exponent result that the $p^{t h}$-power map on $B W_{n}$ is null homotopic.

Proof of Theorem 1.2. Since $\Omega^{2} S^{2 n+1} \stackrel{\nu}{\longrightarrow} B W_{n}$ is an $H$-map, $p \circ \nu \simeq \nu \circ p$. By [CMN2] for $p \geq 5$ and [N] for $p=3$, the $p^{t h}$-power map on $\Omega^{2} S^{2 n+1}$ factors through $S^{2 n-1} \stackrel{E^{2}}{\longrightarrow} \Omega^{2} S^{2 n+1}$. Thus $\nu \circ p$ factors through $\nu \circ E^{2}$, which is null homotopic as $E^{2}$ and $\nu$ are consecutive maps in a homotopy fibration. Hence $p \circ \nu$ is null homotopic. Summarizing, we have maps $\Omega^{2} S^{2 n+1} \stackrel{\nu}{\longrightarrow} B W_{n}$ and $B W_{n} \stackrel{p, *}{\longrightarrow} B W_{n}$ such that $p \circ \nu \simeq * \circ \nu, \Sigma^{2} \nu$ has a right homotopy inverse (by Theorem 2.1), and $B W_{n}$ is an $H$-space. So by Lemma $4.1, p \simeq *$.

\section{ACKNOWLEDGMENT}

The author would like to thank the referee for his helpful comments, particularly for suggesting Lemma 4.1 as a useful abstraction which makes the exposition more focused.

\section{REFERENCES}

[CMN1] F.R. Cohen, J.C. Moore, and J.A. Neisendorfer, Torsion in homotopy groups, Ann. of Math. 109 (1979), 121-168. MR519355 (80e:55024)

[CMN2] F.R. Cohen, J.C. Moore, and J.A. Neisendorfer, The double suspension and exponents of the homotopy groups of spheres, Ann. of Math. 110 (1979), 549-565. MR554384 (81c:55021)

[G1] B. Gray, On the iterated suspension, Topology 27 (1988), 301-310. MR963632 (89h:55016)

[G2] B. Gray, Homotopy commutativity and the EHP sequence, Contemp. Math. 96 (1989), 181-188. MR1022680 (90i:55025)

[H] J.R. Harper, A proof of Gray's conjecture, Contemp. Math. 96 (1989), 189-195. MR1022681 (91b:55013)

[N] J.A. Neisendorfer, 3-Primary exponents, Math. Proc. Camb. Phil. Soc. 90 (1981), 63-83. MR611286 (82e:55026)

[T] S.D. Theriault, Proofs of two conjectures of Gray involving the double suspension, Proc. Amer. Math. Soc. 131 (2003), 2953-2962. MR1974354 (2004h:55006)

Department of Mathematical Sciences, University of Aberdeen, Aberdeen AB24 3UE, UNITED KINGDOM

E-mail address: s.theriault@maths.abdn.ac.uk 